\title{
Age-independent telomere shortening and ion-channel defects in SCD
}

Birendranath Banerjee and M. Prakash Hande

In the Review article by Fyhrquist et al. (The roles of senescence and telomere shortening in cardiovascular disease. Nat. Rev. Cardiol. doi:10.1038/nrcardio.2013.30), ${ }^{1}$ the authors extensively appraise the role in cardiovascular diseases of telomere length homeostasis in response to senescence, oxidative stress, and various other factors. The measurement of leukocyte telomere length to determine telomere size might be an important biomarker to predict cardiac disease outcomes. We agree with most of the conclusions of the authors in this Review and wish to add a new dimension by proposing a possible mechanism by which chronic oxidative stress in the cardiac microenvironment, as a result of ionchannel defects, might cause telomere shortening, which supports the hypothesis of arrhythmia-induced cardiac dysfunction. ${ }^{2}$

We previously reported the direct correlation between telomere length attrition and sudden cardiac death (SCD) using the processes of leukocyte-telomere-length measurement and comparative genomic hybridization. This analysis revealed defects in key ion-channel genes that had a strong correlation with telomere attrition when compared with age-matched controls. ${ }^{2}$ Fyhrquist and colleagues reviewed some work on the role of the mitochondria in cardiovascular disease, but ion channelswhich have a crucial role in maintaining cellular homeostasis, particularly in the heart-were not discussed.

Fyhrquist et al. proposed that the hypothesis of telomere dysfunction might converge with that of mitochondrial dysfunction. They highlighted reports of the presence of increased numbers of senescent cardiomyocytes that express p16, p21, and $\mathrm{p} 53$, and which have short telomeres, in the ageing heart. ${ }^{3}$ In our study, the mean age of individuals who experienced SCD was 24.7 years (range $12-33$ years), and they were compared with controls with a mean age of 23 years (range $21-28$ years). ${ }^{2}$ Severe telomere shortening and ion-channel defects were present in both the cardiomyocytes and peripheral blood lymphocytes of individuals who experienced SCD when compared with age-matched and sex-matched controls. ${ }^{2}$ This phenomenon was observed independently of age. ${ }^{2}$ Post-mortem examinations did not reveal signs of atherosclerotic plaques or cardiomyopathy, which ruled out age-related cardiac dysfunction and degeneration. Therefore, given that in this population, age-induced telomere shortening is well controlled for, the SCD cohort can be used as a model to study telomere attrition.

The association between SCD and arrhythmias is well established. ${ }^{4,5}$ In our study of SCD, we reported deletion of KCNA4 and amplification of RYR2 genes, which actively regulate potassium-channel homeostasis. ${ }^{2}$ These copy number changes in ion-channel genes correlate with telomere regulatory defects in regions such as $3 q 26$ and $18 q 11.2 .^{2,6-8}$ We have also reported that mouse cells that lack telomerase reverse transcriptase are hypersensitive to oxidative stress, and that these mice display rapid upregulation of inflammatory cytokines and increased mortality compared with wildtype mice. ${ }^{9,10}$ We speculate that ion-channel defects and electrical abnormalities of the heart might accelerate telomere shortening by increasing chronic oxidative stress and, thus, cause SCD.

Molecular Stress and Stem Cell Biology Group, School of Biotechnology, Campus XI, KIIT University, Bhubaneswar,
Odisha 751024, India (B. Banerjee). Genome Stability Laboratory, Department of Physiology, Yong Loo Lin School of Medicine, National University of Singapore, MD6, Level 14 South Core, 117597, Singapore (M. P. Hande). Correspondence to: B. Banerjee bnbanerjee@kiitbiotech.ac.in

\section{Acknowledgements}

B. Banerjee is funded by the Department of Biotechnology (DBT), Government of India for his work on molecular stress biology.

\section{Competing interests}

The authors declare no competing interests.

1. Fyhrquist, F., Saijonmaa, O. \& Strandberg, T. The roles of senescence and telomere shortening in cardiovascular disease. Nat. Rev. Cardiol. http://dx.doi.org/10.1038/ nrcardio.2013.30.

2. Banerjee, B. et al. Genomic imbalances in key ion channel genes and telomere shortening in sudden cardiac death victims. Cytogenet. Genome Res. 122, 350-355 (2008).

3. Torella, A. et al. Cardiac stem cell and myocyte aging, heart failure, and insulin-like growth factor-1 overexpression. Circ. Res. 94, 514-524 (2004).

4. Brugada, R. et al. Sudden death associated with short-QT syndrome linked to mutations in HERG. Circulation 109, 30-35 (2004).

5. Oliver, D. et al. Functional conversion between A type and delayed rectifier $\mathrm{K}^{+}$channels by membrane lipids. Science 304, 265-270 (2004).

6. Butler, M. G. et al. Comparison of chromosome telomere integrity in multiple tissues from subjects at different ages. Cancer Genet. Cytogenet. 105, 138-144 (1998).

7. Aviv, A. Genetics of leukocyte telomere length and its role in atherosclerosis. Mutat. Res. 730, 68-74 (2012)

8. Mangino, M. et al. Genome-wide meta-analysis points to CTC1 and ZNF676 as genes regulating telomere homeostasis in humans. Hum. Mol. Genet. 21, 5385-5394 (2012).

9. Newman, J. P. et al. Short dysfunctional telomeres impair the repair of arsenite-induced oxidative damage in mouse cells. J. Cell. Physiol. 214, 796-809 (2008).

10. Bhattacharjee, R. N., Banerjee, B., Akira, S. $\&$ Hande, M. P. Telomere-mediated chromosomal instability triggers TLR4 induced inflammation and death in mice. PLOS ONE 5, e11873 (2010). 